\title{
Short-term effect of laparoscopic assisted total extraperitoneal repair with small-incision for large inguinal hernia in adults.
}

\author{
Yu-huan Wang*, Jiong Fu, Qing-feng Chen, Dong-xu Wang, Wei Jiang, Zheng Chen \\ Department of General Surgery, Feng Hua People's Hospital, Ningbo City, Zhe Jiang Province, PR China
}

\begin{abstract}
Objective: To investigate the short-term efficacy of laparoscopic assisted Total Extraperitoneal Repair (TEP) with small incision for large inguinal hernia in adults, so as to guide the treatment of patients.

Methods: A total of 32 adults with large inguinal hernia treated with laparoscopic assisted TEP from September 2015 to March 2017 were enrolled as the observation group, and from January 2013 to June 2015, 34 cases with large inguinal hernia treated with TEP were enrolled as the control group. The efficacy of operation and recovery, White Blood Count (WBC), C Reactive Protein (CRP) and Visual Analogue Score (VAS), the recurrence rate within 6 months and quality of life between two groups were compared.

Results: Compared with the control group, the operation time in observation group was longer, intraoperative bleeding volume was less, postoperative ambulation time and hospital stays were shorter, the WBC, CRP and VAS at $3 \mathrm{~d}$ after operation were less, the differences were statistically significant $(P<0.05)$. There was no significant difference between two groups in the incidence of complications and recurrence rate within 6 months after operation $(P>0.05)$. Within 6 months after surgery, quality of life in two groups improved significantly $(\mathbf{P}<\mathbf{0 . 0 5})$, but short- term health survey of 36-items $(\mathbf{S F}-36)$ scores were close in two groups before and after surgery $(P>0.05)$.

Conclusion: Laparoscopic assisted TEP with small incision is effective in treatment of large inguinal hernia in adult patients. Compared with the traditional TEP, intraoperative bleeding volume in laparoscopic assisted TEP with small incision is less, postoperative recovery is faster, pain and physiological stress are less.
\end{abstract}

Keywords: Small incision, Laparoscopy, Total extraperitoneal repair, Large inguinal hernia.

Accepted on December 12, 2017

\section{Introduction}

Inguinal hernia, a kind of clinical common disease, refers to the hernia formed by the internal organs of the patient in the abdominal cavity through the defect of the inguinal region, especially in the elderly patients, and the incidence rate at age of $45 \mathrm{y}$ or older is as high as $4 \%$ [1], because of its difficulty in self-healing, the main treatment of large inguinal hernia now is surgical treatment [2]. It is relatively complex to deal with large inguinal hernia, because the defect of abdominal wall tissue and myopectineal orifice area is bigger. In addition, repair of free hernial sac, seal hernial ring and groin are difficult [3]. Totally extraperitoneal inguinal hernia repair (TEP) is one of the standard operations for hernia repair [4], including open and totally chamber microscopically [5], which has been widely applied in the treatment of inguinal hernia, TEP has the advantages of a small incision, fast postoperative recovery etc. [6]. Laparoscopic TEP can repair the normal physiology lacuna in inguinal area, reduce the complications and the recurrence rate, and previous studies confirmed the laparoscopic assisted surgical procedures have a higher application value than traditional surgery [7]. But in previous studies, the experience was mainly of senior hospitals, and which lacked of comparative analysis of the large inguinal hernia. While our study took the primary hospitals as example, to comparatively analyse the application value of laparoscopic assisted TEP operation with small incision for large inguinal hernia. The report is as follows.

\section{Materials and Methods}

\section{General information}

In our hospital, from September 2015 to March 2017, 32 adult patients with inguinal hernia accepted laparoscopic assisted TEP with small incision and were regarded as the observation group, and from January 2013 to June 2015, 34 patients accepted TEP were recruited as control group, and the casecontrol research was carried out. This study has obtained the approval of Ethics Committee in our hospital. The observation group included males aged 38 74 y old, average aged (48.41 \pm $11.52 \mathrm{y}$ old); disease course of $2 \sim 8 \mathrm{y}$, average disease course of $(4.81 \pm 1.37 \mathrm{y})$; including 5 cases of direct hernia, 20 cases of indirect hernia, 7 cases of composite hernia; diameter of defect 
in hernia ring of $(3.82 \pm 0.71 \mathrm{~cm})$, and the hernia perimeter $(25.82 \pm 3.68 \mathrm{~cm})$. The control group included males aged $34 \sim 75$ y old, average aged (49.18 \pm 10.85 y old); disease course of $2 \sim 11 \mathrm{y}$, average disease course of $(4.76 \pm 1.24 \mathrm{y})$; including 4 cases of direct hernia, 22 cases of indirect hernia, 8 cases of composite hernia, diameter of defect in hernia ring of $(3.98 \pm 0.71 \mathrm{~cm})$, the hernia perimeter $(25.12 \pm 3.17 \mathrm{~cm})$. The differences of two groups on gender, age, course of disease and so on were not statistically significant $(\mathrm{P}>0.05)$.

\section{Inclusion and exclusion criteria}

Inclusion criteria: (1) Aged $>18$ y old; (2) Meeting the criteria of "Evidence-Based Hernia Treatment in Adults" [8], as well as surgical indications of TEP; (3) Refer to the relevant criteria in "Guideline of diagnosis and treatment of inguinal hernia in adults (2014 edition)" [9], patients with type III, the diameter of hernia ring of $\geq 3 \mathrm{~cm}$ and hernia perimeter of $\geq 20 \mathrm{~cm}$; (4) Unilateral inguinal hernia; (5) Patients were informed by this study and signed the informed consent. Exclusion criteria: (1) Previous history of abdominal surgery; (2) Severe cardiopulmonary dysfunction or coagulation dysfunction; (3) Patients with recurrent hernia.

\section{Surgical procedures}

Surgery was performed by the same surgical team in two groups. The observation group with general anesthesia adopted laparoscopic assisted TEP with small incision, all the patients underwent surgery with the supine position. A small incision of $1.0 \mathrm{~cm}$ was made at $1.0 \mathrm{~cm}$ below the umbilicus, the first trocar was placed in front of the rectus sheath. Preliminary, peritoneal space was created by laparoscopic pushing method. The second and third trocar adopted midline method respectively, which the puncture point was made between the site of $1 / 3$ up and $1 / 3$ down in the center of umbilicus and pubic symphysis and the preperitoneal space was opened. Next, the Bogros and Retzius space were separated; a horizontal incision about 2-3 $\mathrm{cm}$ was made in the internal inguinal ring, and the skin, subcutaneous tissue, aponeurosis of obliquus externus abdominis in turn were dissected. The obliquus internus abdominis and musculus trasversus abdominis were dissected bluntly, the transverse abdominal velum were opened to enter the preperitoneal space. The hernia sac were sutured after separated free hernia sac at high level, the $6 \mathrm{~cm}$ of the rear of the spermatic cord blood vessels and vas deferens in inner level were separated and completed, so that the spermatic cord abdominal wall was finished. The horizontal incisions in turn were closed, further expansion was done under laparoscopic and the preperitoneal space was confirmed step by step. The preperitoneal space to the pubic symphysis was separated cross the midline, on the outside to iliopsoas and anterior superior iliac spine, up to conjoined tendons about $2 \mathrm{~cm}$, down to pectineal ligament inside about $2.0 \mathrm{~cm}$, and outside down to the level of spermatic abdominal wall to the vas deferens and spermatic cord blood vessel bifurcation about $6 \mathrm{~cm}$. Finally, preperiotneal repair was finished under the direct view of laparoscopic. The entire myopectineal orifice was covered by
BARD 3D Max large mesh-plug, fixation of the Compont glue was used. Drainage tube was placed in preperitoneum, and conventional treatment of puncturing was done after laparoscope. The control group adopted TEP with spinal epidural anesthesia, all the patients underwent surgery with the supine position. A centre incision in the hypogastrium was made, the rectus sheath was dissected, the rectus abdominis was opened to expose the fascia transversalis, and the transversus abdominis plane was dissected. On this level, the lateral downward and outside was separated for revealing the blood vessels under abdominal wall and iliopubic tract outside and pectineal ligament downward. After finished the separation, the hernia sac, spermatic cord abdominal wall and placement of mesh-plug were implanted according to the schemes in the observation group. Finally, the incisions were sutured in turn.

\section{Observational indexes}

(1) General surgical condition included the operation time, intraoperative blood volume, postoperative ambulation time, and hospital stays. (2) Postoperative complications mainly included hematoma or seroma, infection, groin pain. (3) Postoperative stress and pain. At $1 \mathrm{~d}$ before operation or $3 \mathrm{~d}$ after operation, LH755 automatic blood analyzer (American beckman coulter company) was used to detect peripheral blood leukocyte count (WBC), C reactive protein (CRP) was detected by using enzyme-linked immunosorbent experiments (purchased from Shanghai Xinfan Biological Technology CO., LTD). And the degree of pain were analysed by Visual Analogue Score (VAS) at $1 \mathrm{~d}$ before operation or $3 \mathrm{~d}$ after operation. (4) Within 6 months of follow-up, patients would return visit to accept the clinical examination in hospital, then recurrence of patients were observed. (5) 36-items short form health survey (SF-36) was adopted to evaluate patients' quality of life at 1-3 d before operation and 6 months after discharged, the scale includes 8 dimensions, for example, physical function, physiological function, body pain, general health, energy, social function, emotional function, mental health, the higher the score in each field, the better the quality of life in patients, and the Cronbach 's alpha coefficients in SF-36 is above 0.70 [10].

\section{Statistical methods}

SPSS19.0 software was used to analyse the data, measurement data were expressed as $(\overline{\mathrm{x}} \pm \mathrm{s})$, the independent sample was analysed by t-test, the counting data were expressed by percentage, analysed by the chi-square test. $\mathrm{P}<0.05$ was considered the difference was statistically significant.

\section{Results}

\section{Comparisons of surgical effects between two groups and the display of operation cases}

All patients in two groups received the surgery successfully, and were well-healed and discharged normally from hospital. Compared with control group, the operating time of 
Short-term effect of laparoscopic assisted total extraperitoneal repair with small-incision for large inguinal hernia in adults

observation group was longer, intraoperative blood volume was less, postoperative ambulation time and hospital stays were shorter, the differences were statistically significant $(\mathrm{P}<0.05)$, the results are shown in Table 1 , and the display of operation cases are shown in Figures 1-3.

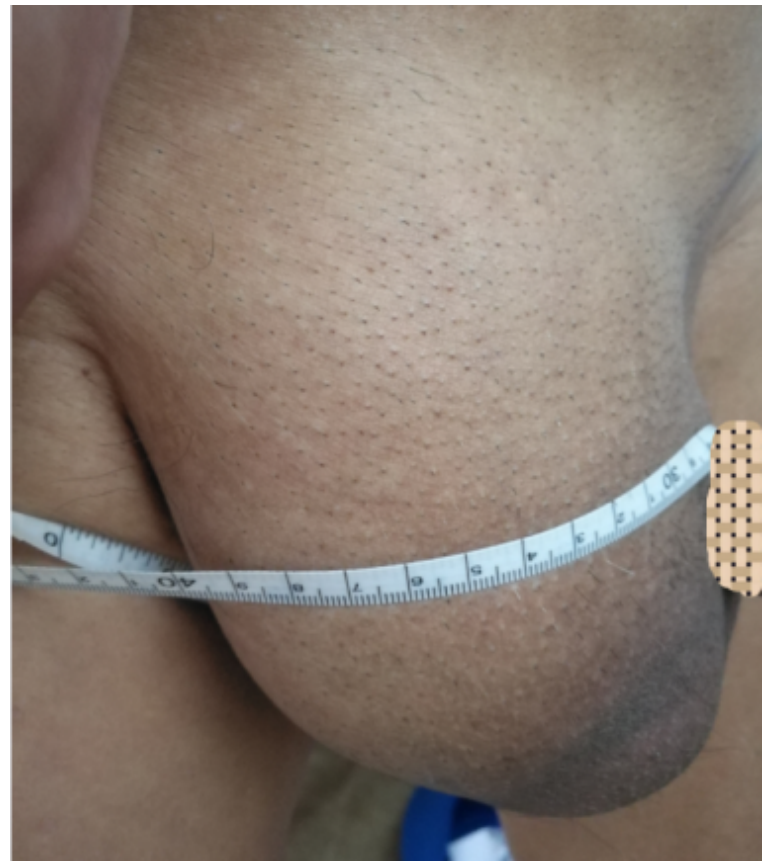

Figure 1. Display of right large hernia in preoperation.

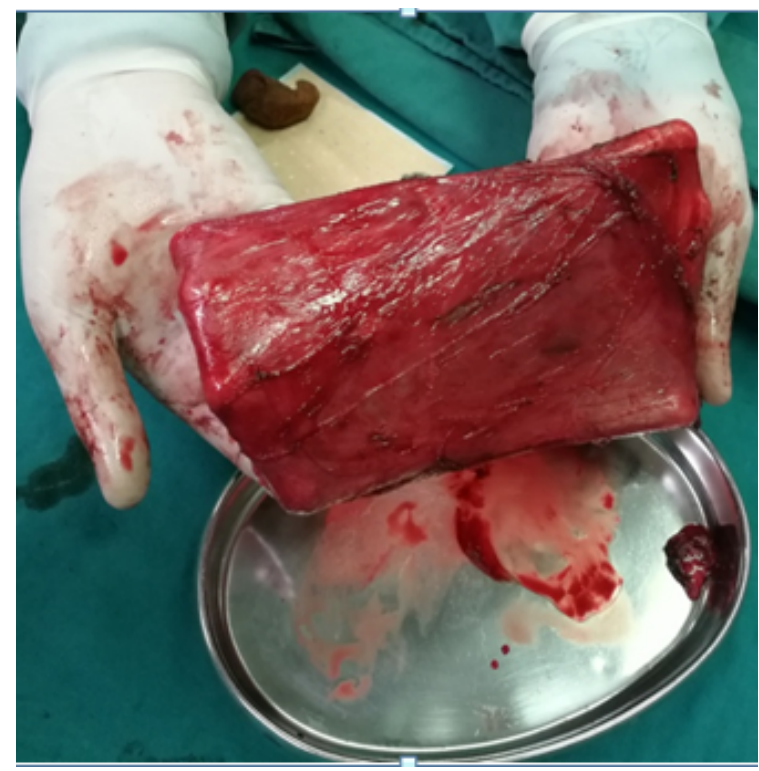

Figure 2. Removed hernia tissue in intraoperation.

\section{Comparisons of incidence of postoperative complications between two groups}

The total incidence was very lower in the observation group than that in the control group, but there was no statistically significant difference $(\mathrm{P}>0.05)$, the results are shown in Table 2.

\section{Comparisons of WBC, CRP and VAS of two groups at 3 d after operation}

In two groups, the differences in WBC, CRP level and VAS were no statistically significant $(\mathrm{P}>0.05)$, the above indicators were significantly increased at $3 \mathrm{~d}$ after operation $(\mathrm{P}<0.05)$, and the WBC, CRP and VAS of observation group were significantly less than those of the control group $(\mathrm{P}<0.05)$, the results are shown in Table 3.

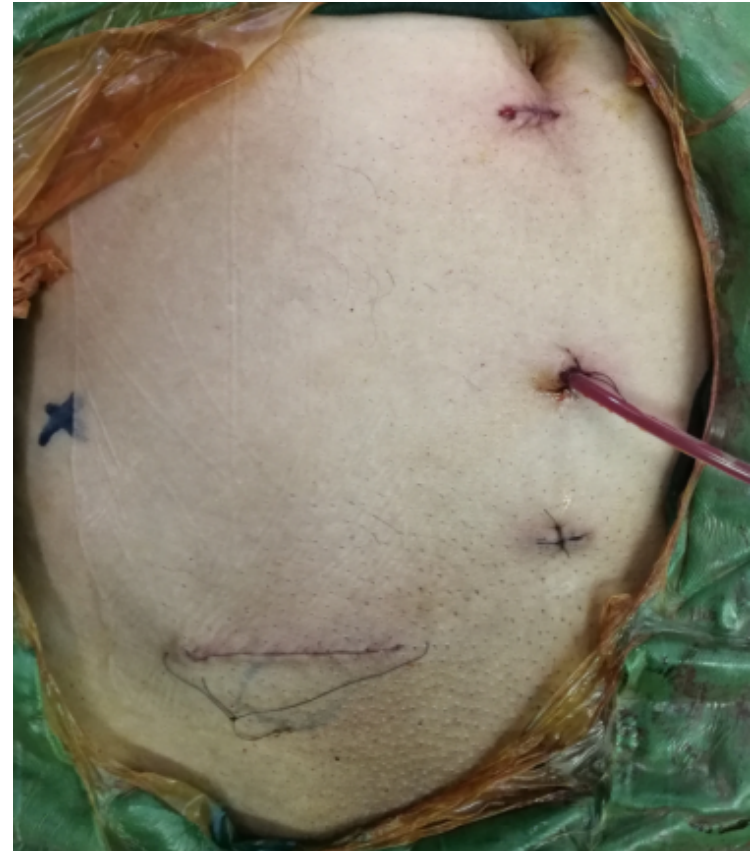

Figure 3. Display of mini-incision in postoperation.

\section{Comparisons of recurrence and quality of life in patients of two groups}

Within 6 months of follow-up, the observation group had 0 case of recurrence, the control group had 1 case of recurrence, there was no statistically significant difference in the two groups $(\mathrm{P}=0.328)$. Of two groups, the scores of $\mathrm{SF}-36$ scale in every dimensions showed that there was no statistically significant difference before operation and within 6 months after operation $(\mathrm{P}>0.05)$, and within 6 months after discharged from hospital, the scores of SF-36 scale in every dimensions were higher, the differences were statistically significant $(\mathrm{P}<0.05)$, the results are shown in Table 4 .

\section{Discussion}

Large inguinal hernia is found mostly in males [11], and has relatively difficulty for treatment, this research showed that the TEP has reliable therapeutic effect on large inguinal hernia. Patients in two groups received surgical procedures, and were well-healed, besides, within 6 months of follow-up there was 1 case of recurrence in the control group, which indicated that the TEP is a reliable solution for the treatment of adult patient with large inguinal hernia. Although, there is rare application value of pertinent analysis in TEP of large inguinal hernia, according to the clinical criteria, large inguinal hernia belongs 
to type III of inguinal hernia, part of the reports included the type III patients, and the results can confirm the TEP's advantages. For example, Shuyong et al. [12] reported that a total of 34 patients were enrolled, in the report of Tongsheng et al. [13], totally 41 patients with type III were included, and the results are similar with this study.

Laparoscopic Transabdominal Preperitoneal Hernia Repair (TAPP) have similar efficacy with TEP, TAPP was operated in abdominal cavity, which may resulted in patients with more hospital stays and long recovery [14]. However, The advantages of TEP are that surgeons do not operate in the abdominal cavity, which avoided the possibility of injuring organs, port-site hernia, intestinal adhesion due to abdominal intranet piece repairing and abdominal preperitoneal patch in the abdominal cavity, less postoperative pain, mesh-plug hernia repair without structure and fixation at the same time, so that postoperative complications can be reduced [15-17]. TEP would be completed by laparoscopic or traditional surgery, this research adopted the laparoscopic (not abdominal cavity but totally peritoneal) assisted TEP operation with small incision, by means of laparoscopic TEP combined with different pathways. TEP isn't only in the abdominal cavity with the advantages of less postoperative pain, but also the difficulty of separating large hernia sac and spermatic cord abdominal wall can be alleviated and it can avoid the bleeding and injured spermatic cord for reducing the surgical risks. Intraoperative bleeding volume of observation group was less than that of the control group, postoperative ambulation time and hospital stays were shorter than those of the control group, and the results are consistent with Jinson [18] and it indicated the fewer traumas in patients with this surgery. At the same time, postoperative pain except other complications and recurrence rate within 6 months in two groups were less, which is consistent with the report of Ming et al. [19], indicating that the laparoscopic surgery can maintain the superiority of less injured organs and lower recurrence rate. But this study found that operation time in observation group was longer than that in the control group, and the main reasons should be found that this operation cannot be completed by using routine equipment under direct view, therefore, with the long-term accumulation of learning experience in primary hospital, the operation time of the surgery can be gradually shortened to meet the clinical demand, which accorded with Lidan et al. [20]. Both CRP and WBC can directly reflect the body's inflammatory and trauma situation $[21,22]$, and this study showed that peripheral blood CRP and WBC, as well as VAS scores in the observation group were significantly less than those in the control group at $3 \mathrm{~d}$ after operation, which confirmed the surgical trauma of patients is less, meanwhile the pain of patients is reduced. This study found that before surgery or at 6 months after surgery, the quality of life in two groups was close, and the results explained two surgical schemes can effectively guarantee the quality of life of patients.

There are two key points about total peritoneal operation as follows: first, the creation and maintenance of the preperitoneal space; second, the standard operation of preventing the recurrence. The specific points mainly including: (1) The bleeding caused by injured blood vessels such as abdominal blood vessels, pubic venous plexus, dangerous calot triangle, corona mortis should be strictly avoided in operation, because bleeding cause the indistinct view, and it is not easy to hold when aspirator is removing extra blood in the preperitoneal space; (2) The small stripped incision was opened to close the incision after finished the spermatic cord abdominal wall, for maintaining the leakproofness of the preperitoneal space; (3) Freeing hernia sac should be paid attention, and patch needs to be large enough to cover the whole myopectineal orifice. Due to the large defect of the hernia, it is necessary to be fixed by using the compont glue.

In summary, laparoscopic assisted TEP operation with small incision gains curative effect on large inguinal hernia for adults, reduces intraoperative bleeding, accelerates the recovery after operation, and alleviates the patient's pain and physiological stress. But less number of included cases was the shortage in this study, we still need to pay attention to larger sample size with randomized-design in next study.

Table 1. Comparisons of surgical efficacy of two group $(\bar{x} \pm s)$.

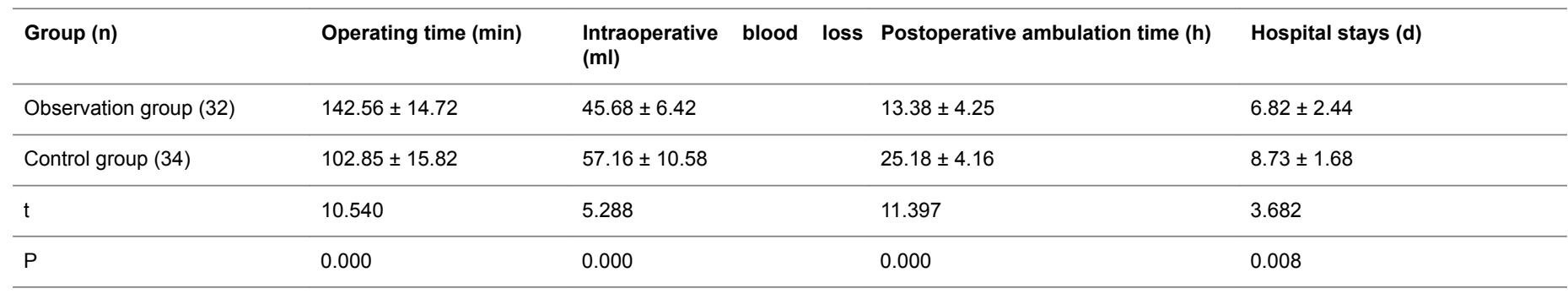

Table 2. The comparisons of complications occurred in the two groups (n (\%)).

\begin{tabular}{|c|c|c|c|c|c|}
\hline Group (n) & Hydrocele & Hematoma or seroma & Wound infection & Wound disruption & Total \\
\hline Observation group (32) & $0(0.00)$ & $1(3.13)$ & $1(3.13)$ & $0(0.00)$ & $2(6.25)$ \\
\hline
\end{tabular}


Short-term effect of laparoscopic assisted total extraperitoneal repair with small-incision for large inguinal hernia in adults

\begin{tabular}{|c|c|c|c|c|c|}
\hline Control group (34) & $1(2.94)$ & $1(2.94)$ & $0(0.00)$ & $1(2.94)$ & $3(8.82)$ \\
\hline$x^{2}$ & & & & & 0.156 \\
\hline $\mathrm{P}$ & & & & & 0.693 \\
\hline
\end{tabular}

Table 3. Comparisons of WBC and CRP of two groups at 3 d after operation $(\bar{x} \pm s)$.

\begin{tabular}{|c|c|c|c|c|c|c|}
\hline \multirow[t]{2}{*}{ Group (n) } & \multicolumn{2}{|l|}{ WBC $\left(\times 10^{9} / L\right)$} & \multicolumn{2}{|l|}{ CRP (mg/L) } & \multicolumn{2}{|l|}{ VAS } \\
\hline & $\begin{array}{l}\text { At } 1 \text { d before } \\
\text { operation }\end{array}$ & $\begin{array}{l}\text { At } 3 \text { d after } \\
\text { operation }\end{array}$ & $\begin{array}{l}\text { At } 1 \text { d before } \\
\text { operation }\end{array}$ & $\begin{array}{l}\text { At } \quad 3 \\
\text { operation }\end{array}$ & $\begin{array}{l}\text { At } 1 \text { d before } \\
\text { operation }\end{array}$ & $\begin{array}{l}\text { At } \quad 3 \\
\text { operation }\end{array}$ \\
\hline Observation group (32) & $5.48 \pm 1.61$ & $6.17 \pm 1.53^{*}$ & $0.74 \pm 0.38$ & $6.64 \pm 1.25^{*}$ & $4.56 \pm 1.12$ & $2.03 \pm 0.49^{*}$ \\
\hline Control group (34) & $5.53 \pm 1.43$ & $9.65 \pm 1.17^{*}$ & $0.81 \pm 0.41$ & $10.72 \pm 1.68^{*}$ & $4.81 \pm 1.23$ & $3.01 \pm 0.47^{*}$ \\
\hline$t$ & 0.134 & 10.417 & 0.718 & 11.138 & 0.862 & 8.293 \\
\hline$P$ & 0.894 & 0.000 & 0.475 & 0.000 & 0.392 & 0.000 \\
\hline
\end{tabular}

Note: ${ }^{*}$ compared with $1 \mathrm{~d}$ before operation, $\mathrm{P}<0.05$.

Table 4. Comparisons of quality of life in patients of two groups $(\bar{x} \pm s)$.

\begin{tabular}{|c|c|c|c|c|c|c|c|c|}
\hline Items & $\begin{array}{l}\text { Physical } \\
\text { function }\end{array}$ & $\begin{array}{l}\text { Physiological } \\
\text { function }\end{array}$ & Body pain & $\begin{array}{l}\text { General } \\
\text { health }\end{array}$ & Energy & $\begin{array}{l}\text { Social } \\
\text { function }\end{array}$ & $\begin{array}{l}\text { Emotional } \\
\text { function }\end{array}$ & Mental health \\
\hline \multicolumn{9}{|l|}{ Observation group (32) } \\
\hline At $1 \mathrm{~d}$ after operation & $45.47 \pm 6.14$ & $62.25 \pm 7.79$ & $23.97 \pm 6.52$ & $61.19 \pm 7.78$ & $66.84 \pm 7.82$ & $64.63 \pm 8.69$ & $67.14 \pm 10.25$ & $70.81 \pm 6.79$ \\
\hline At 6 months after discharged & $90.19 \pm 5.43$ & $95.03 \pm 3.13$ & $39.22 \pm 2.43$ & $93.81 \pm 3.63$ & $91.88 \pm 3.96$ & $94.66 \pm 2.87$ & $93.36 \pm 12.18$ & $92.47 \pm 4.70$ \\
\hline $\mathrm{t}$ & 28.464 & 21.683 & 12.000 & 25.774 & 15.899 & 17.604 & 9.883 & 15.355 \\
\hline$P$ & 0.000 & 0.000 & 0.000 & 0.000 & 0.000 & 0.000 & 0.000 & 0.000 \\
\hline \multicolumn{9}{|l|}{ Control group (34) } \\
\hline At $1 \mathrm{~d}$ after operation & $45.38 \pm 5.60$ & $65.44 \pm 6.82$ & $26.47 \pm 6.36$ & $59.32 \pm 6.82$ & $68.97 \pm 9.83$ & $62.50 \pm 6.03$ & $68.36 \pm 10.58$ & $72.44 \pm 8.35$ \\
\hline At 6 months after discharged & $90.94 \pm 5.15$ & $95.73 \pm 2.60$ & $39.68 \pm 3.24$ & $93.79 \pm 3.87$ & $93.03 \pm 4.04$ & $93.76 \pm 4.67$ & $80.14 \pm 11.51$ & $92.97 \pm 6.69$ \\
\hline $\mathrm{t}$ & 41.745 & 25.424 & 9.814 & 25.893 & 13.270 & 26.209 & 4.521 & 10.210 \\
\hline$P$ & 0.000 & 0.000 & 0.000 & 0.000 & 0.000 & 0.000 & 0.000 & 0.000 \\
\hline
\end{tabular}

\section{Fund Project}

Fenghua social development of science and technology project No: 20162708.

\section{References}

1. Alimu A, Pierdiwasi A. A study on the prevalence of inguinal hernia in Mukarm artists in xinjiang. Chin J Gen Surg 2013; 28: 628-629.

2. de Goede B, Timmermans L, van Kempen BJ. Risk factors for inguinal hernia in middle-aged and elderly men: results from the Rotterdam Study. Surgery 2015; 157: 540-546.

3. Cavalli M, Biondi A, Bruni PG. Giant inguinal hernia: the challenging hug technique. Hernia 2015; 19: 775-783.

4. Kockerling F, Jacob DA, Chowbey P. Totally extraperitoneal repair of inguinal hernias: some remarks on technical details. Langenbecks Arch Surg 2013; 398: 481-482.
5. Wakasugi M, Masuzawa T, Tei M. Single-incision totally extraperitoneal inguinal hernia repair: our initial 100 cases and comparison with conventional three-port laparoscopic totally extraperitoneal inguinal hernia repair. Surg Today 2015; 45: 606-610.

6. Bobo Z, Nan W, Qin Q. Meta-analysis of randomized controlled trials comparing Lichtenstein and totally extraperitoneal laparoscopic hernioplasty in treatment of inguinal hernias. J Surg Res 2014; 192: 409-420.

7. Hu Minggao, Li Xiangguo, Guo Biao. Comparison study of extraperitoneal inguinal herniorrhaphy with open and laparoscopic technique. Chin J Bases Clin General Surg 2014; 21: 1431-1435.

8. Berger D. Evidence-based hernia treatment in adults. Dtsch Arztebl Int 2016; 113: 150-158.

9. Hernia and abdominal wall surgery group of Chinese medical organization of surgery branch. Guideline of 
diagnosis and treatment of inguinal hernia in adults (2014 edition). Chinese J Surg 2014; 52: 484-486.

10. Jia-qiang L, Min L, Juying Z. Studying on the reliable and validity of scale of SF-36 to determine the life quality of patients with sciatica caused by lumbar disc herniation. Chinese Health Serv Manag 2013; 30: 144-145.

11. Hajong R. One stage repair of giant inguinal hernia. J Coll Physicians Surg Pak 2014; 24: 218.

12. Shuyong G, Yingling S, Zongheng Z. The comparative analysis of the efficacy of TEP and open-tension hernia repair for adult inguinal hernia. Chin J Curr Adv Gen Surg 2015; 18: 481-483.

13. Wang T, Ding L, Zhao A. Comparative analysis of laparoscopic total extraperitoneal repair versus preperitoneal inguinal hernia repair via midline incision. Chin J Min Inv Surg 2016; 16: 118-121.

14. Sheng-ping C, Chun-wei G. Clinical evaluation of TEP and TAPP in treatment of inguinal hernia. Chinese J Curr Adv Gene Surg 2015; 18: 736-737.

15. Lin $\mathrm{CD}$, Wu CH, Liu YB. Feasibility and safety of laparoendoscopic single-site surgery of total extraperitoneal inguinal hernia repair after previous open groin hernia repair: a comparative study. Surg Endosc 2016; 30: 2086-2089.

16. Saka R, Okuyama H, Sasaki T. Safety and efficacy of laparoscopic percutaneous extraperitoneal closure for inguinal hernias and hydroceles in children: a comparison with traditional open repair. J Laparoendosc Adv Surg Tech A $2014 ; 24: 55-58$
17. Kockerling F, Bittner R, Jacob DA. TEP versus TAPP: comparison of the perioperative outcome in 17,587 patients with a primary unilateral inguinal hernia. Surg Endosc 2015; 29: 3750-3760.

18. Li J. Application of laparoscopic TEP and open abdominal hernia repair in primary hospital. Chin J Min Inv Surg 2016; 19: 351-354.

19. Jiang M, Jiang Y, Wang J. Comparison of total extraperitoneal and transabdominal preperitoneal in laparoscoic inguinal herniorrhaphy. Chin J Bases Clin General Surg 2013; 20: 1391-1395.

20. Zhu L, Wu L, Wang H. Learning curve analysis of totally extraperitoneal prosthesis. Anhui Med Pharm 2013; 17: 1322-1324.

21. Noble F, Curtis NJ, Underwood TJ. C-reactive protein 2 days after laparoscopic gastric bypass surgery reliably indicates leaks and moderately predicts morbidity. J Gastrointest Surg 2013; 17: 844-845.

22. Chen $X$. Comparison of the efficacy and safety of three types of patching in inguinal hernia. Chin J Hernia Abdom Wall Surg 2015; 9: 35-38.

\section{*Correspondence to}

Yu-huan Wang

Department of General Surgery

Feng Hua People's Hospital

PR China 